\title{
Dynamical noncommutativity
}

\author{
M. Gomes, V.G. Kupriyanov, A.J. da Silva \\ Instituto de Física, Universidade de São Paulo, Brazil
}

August 23, 2021

\begin{abstract}
The model of dynamical noncommutativity is proposed. The system consists of two interrelated parts. The first of them describes the physical degrees of freedom with coordinates $q^{1}, q^{2}$, the second one corresponds to the noncommutativity $\eta$ which has a proper dynamics. After quantization the commutator of two physical coordinates is proportional to the function of $\eta$. The interesting feature of our model is the dependence of nonlocality on the energy of the system. The more the energy, the more the nonlocality. The leading contribution is due to the mode of noncommutativity, however, the physical degrees of freedom also contribute in nonlocality in higher orders in $\theta$.
\end{abstract}

\section{Introduction}

It is generally believed, that due to the effects of the gravitational backreaction the classical background space-time is nonlocal on very small distances, those comparable with the Planck scale [1]. Since noncommutativity usually leads to nonlocality, noncommutative geometry was proposed as a mathematical tool to systematically incorporate nonlocality in physics. The simplest approximation to a noncommutative space-time is a flat canonical noncommutativity, which can be realized by the coordinate operators $\hat{q}^{\mu}$ satisfying commutation relations $\left[\hat{q}^{\mu}, \hat{q}^{\nu}\right]=$ $i \theta^{\mu \nu}$, where $\theta^{\mu \nu}$ is an antisymmetric constant matrix. Thus, quantum field theory, $\mathrm{QFT}$, and quantum mechanics, QM, on flat noncommutative spaces have recently been extensively studied, see e.g. [2] and [3] for reviews on noncommutativity in QFT and QM, respectively.

However, it is not reasonable to suppose that the noncommutativity and hence nonlocality is the same in the whole universe. It can depend on the point of the space-time, see e.g., [4] and references therein. A model of position-dependent 
noncommutativity in quantum mechanics, involving the complete algebra of noncommutative coordinates:

$$
\left[\hat{q}^{\mu}, \hat{q}^{\nu}\right]=i \omega^{\mu \nu}(\hat{q})
$$

where $\omega^{\mu \nu}$ is some given matrix-valued function, and corresponding momenta was recently proposed in [5].

To precisely specify $\omega^{\mu \nu}(q)$, physical considerations must be taken into account. Strong magnetic fields, quantum oscillations associated to pair creation and strings dynamics are just some causes that may lead to noncommutativity. A basic question here is to uncover the mechanism leading to noncommutativity. One of the possibilities is to suppose that noncommutativity has its own degrees of freedom which interact in some way with the physical degrees of freedom of the system. Since noncommutativity $\omega^{\mu \nu}(q)$ is a field it has an infinite number of degrees of freedom and its evolution should be described by a field equation of motion. To simplify our considerations and to better understand what happens, we suppose that noncommutativity has only one degree of freedom, embodied by the variable $\eta$. We also suppose that the physical system under study is described by two coordinates $q^{1}$ and $q^{2}$. In this situation, the most general form of the commutation relations is

$$
\left[\hat{q}^{i}, \hat{q}^{j}\right]=i \theta f(\hat{\eta}) \varepsilon^{i j}
$$

where $f$ is some function of the indicated argument and $\theta$ is a parameter which gives the strength of the noncommutativity. By itself, Equation (2) provides the main effect of the interaction between $\eta$ and $q_{1}, q_{2}$. This type of commutation relation we call dynamical noncommutativity.

In this work, we consider a classical model which after quantization reproduces the dynamical noncommutativity (2) and discuss some of its properties. An interesting feature of the system in question is the dependence of the nonlocality on the energy of the system. The leading contribution comes from the modes associtated to the variable $\eta$, but the physical degrees of freedom $q_{1}$ and $q_{2}$ also contribute to nonlocality in higher orders.

\section{The classical model and quantization}

Consider the following singular Lagrangian1:

$$
\begin{aligned}
L & =L_{q}+L_{r}+L_{i n t}, \\
L_{q} & =v_{i} \dot{q}^{i}-h(v, q), \quad L_{\eta}=\frac{\dot{\eta}^{2}}{2}-V(\eta), L_{i n t}=\frac{\theta}{2} f(\eta) \varepsilon^{i j} v_{i} \dot{v}_{j},
\end{aligned}
$$

\footnotetext{
${ }^{1}$ For simplicity we consider two-dimensional case, $q^{i}=\left(q^{1}, q^{2}\right)$, however, the generalization to the $n$-dimensional case is straightforward.
} 
here $h(v, q)$ and $V(\eta)$ are some given functions and $q,, v, \eta$ are an independent variables 2 It consists of three parts: $L_{q}$ and $L_{\eta}$ describe dynamics of two subsystems with coordinates $q^{1}, q^{2}$ and $\eta$ correspondingly, and $L_{\text {int }}$ describes the interaction between these two subsystems. Notice that first order actions containing a term similar to $L_{\text {int }}$ appear in the context of the Landau problem in the limit of strong magnetic field or small electron mass [6] and lead to noncommutativity between the coordinates $q^{i}$ after quantization, see e.g. [7]. Actually, $L_{i n t}$ is the minimal interaction needed to produce the desired commutation relation (2).

To proceed to the Hamiltonian formulation, we introduce conjugate momenta according to the rules

$$
p_{i}=\frac{\partial L}{\partial \dot{q}^{i}}, \quad \pi_{i}=\frac{\partial L}{\partial \dot{v}^{i}}, \quad p_{\eta}=\frac{\partial L}{\partial \dot{\eta}} .
$$

They are considered as algebraic equations for determining the velocities $\dot{q}^{i}, \dot{v}^{i}, \dot{\eta}$. Since the Lagrangian (3) is linear in $\dot{q}^{i}$ and $\dot{v}^{i}$, the first two equations of (4) become primary constraints in the Hamiltonian formalism:

$$
G_{i}=p_{i}-v_{i}=0, T_{i}=\pi_{i}+\frac{\theta}{2} f(\eta) \varepsilon^{i j} v_{j}=0 .
$$

The total Hamiltonian of the theory is:

$$
H^{(1)}=H+\lambda_{p}^{i} G_{i}+\lambda_{\pi}^{i} T_{i}, \quad H=h(p, q)+\frac{p_{\eta}^{2}}{2}+V(\eta)
$$

where $\lambda_{p}^{i}$ and $\lambda_{\pi}^{i}$ are Lagrangian multipliers. In Dirac's terminology (15) are second-class constraints. Thus, from the consistency conditions for the primary constraints

$$
\dot{\Phi}=\left\{\Phi, H^{(1)}\right\}=0,
$$

where $\Phi=(G, T)$, one can fix all the $\lambda$-s. Secondary constraints do not appear. The Hamiltonian equations are

$$
\dot{\chi}=\{\chi, H\}_{D(\Phi)}, \Phi=0
$$

where $\chi=\left(q, v, \eta, p, \pi, p_{\eta}\right)$ and $\{\cdot, \cdot\}_{D(\Phi)}$ are Dirac brackets with respect to second-class constraints (15).

\footnotetext{
${ }^{2}$ One can also consider the situation when $v^{i}$ are velocities, $v^{i}=\dot{q}^{i}$, i.e., a model with higher derivatives, similar to the one proposed in $[8$. This model has some difficulties with the physical interpretation of the additional degrees of freedom, also, its quantum spectrum is not bounded from below 9 .
} 
Since the effective Hamiltonian $H$ depends only on the coordinates $q^{i}, \eta$ and momenta $p_{i}, p_{\eta}$, we write the Dirac brackets only between these variables:

$$
\begin{aligned}
& \left\{q^{i}, q^{j}\right\}_{D(\Phi)}=f(\eta) \theta \varepsilon^{i j}, \quad\left\{q^{i}, p_{j}\right\}_{D(\Phi)}=\delta_{j}^{i} \\
& \left\{q^{i}, p_{\eta}\right\}_{D(\Phi)}=-\frac{\theta}{2} f^{\prime}(\eta) \varepsilon^{i j} p_{j}, \quad\left\{\eta, p_{\eta}\right\}_{D(\Phi)}=1 \\
& \left\{\eta, q^{i}\right\}_{D(\Phi)}=\left\{\eta, p_{i}\right\}_{D(\Phi)}=\left\{p_{\eta}, p_{i}\right\}_{D(\Phi)}=\left\{p_{i}, p_{j}\right\}_{D(\Phi)}=0 .
\end{aligned}
$$

After quantization, these Dirac brackets will determine the commutation relations between operators of coordinates $\hat{q}^{i}, \hat{\eta}$ and the corresponding operators of momenta $\hat{p}_{i}, \hat{p}_{\eta}$ :

$$
\begin{aligned}
& {\left[\hat{q}^{i}, \hat{q}^{j}\right]=i \theta f(\hat{\eta}) \varepsilon^{i j}, \quad\left[\hat{q}^{i}, \hat{p}_{j}\right]=i \delta_{j}^{i},} \\
& {\left[\hat{q}^{i}, \hat{p}_{\eta}\right]=-\frac{i \theta}{2} f^{\prime}(\hat{\eta}) \varepsilon^{i j} \hat{p}_{j}, \quad\left[\hat{\eta}, \hat{p}_{\eta}\right]=i} \\
& {\left[\hat{\eta}, \hat{q}^{i}\right]=\left[\hat{\eta}, \hat{p}_{i}\right]=\left[\hat{p}_{\eta}, \hat{p}_{i}\right]=\left[\hat{p}_{i}, \hat{p}_{j}\right]=0 .}
\end{aligned}
$$

That is, dynamical noncommutativity (2) can be reproduced by the quantization of the system with Lagrangian (3). The quantum Hamiltonian is

$$
\hat{H}=h(\hat{p}, \hat{q})+\frac{\hat{p}_{\eta}^{2}}{2}+V(\hat{\eta})
$$

where some ordering is implicitly assumed.

To construct representation of the operatorial algebra 3 (10) let us introduce new operators

$$
\hat{x}^{i}=\hat{q}^{i}+\frac{\theta}{2} f(\hat{\eta}) \varepsilon^{i j} \hat{p}_{j}
$$

It is easy to see that operators $\hat{x}^{i}, \hat{\eta}, \hat{p}_{i}, \hat{p}_{\eta}$ obey canonical commutation relations:

$$
\begin{aligned}
& {\left[\hat{x}^{i}, \hat{p}_{j}\right]=i \delta_{j}^{i},\left[\hat{\eta}, \hat{p}_{\eta}\right]=i,} \\
& {\left[\hat{x}^{i}, \hat{x}^{i}\right]=\left[\hat{\eta}, \hat{x}^{i}\right]=\left[\hat{x}^{i}, \hat{p}_{\eta}\right]=0,} \\
& {\left[\hat{\eta}, \hat{p}_{i}\right]=\left[\hat{p}_{i}, \hat{p}_{j}\right]=\left[\hat{p}_{\eta}, \hat{p}_{i}\right]=0 .}
\end{aligned}
$$

Thus, similarly to a Bopp shift [11], we pass to the new set of operators $\hat{x}^{i}, \hat{\eta}, \hat{p}_{i}, \hat{p}_{\eta}$ with canonical commutation relations (13). The quantum Hamiltonian (11) in the new variables will take the form

$$
\hat{H}=h\left(\hat{p}_{i}, \hat{x}^{i}-\frac{\theta}{2} f(\hat{\eta}) \varepsilon^{i j} \hat{p}_{j}\right)+\frac{\hat{p}_{\eta}^{2}}{2}+V(\hat{\eta}) .
$$

\footnotetext{
${ }^{3}$ Note that in the general case, the polydifferential representation of the operatorial algebra defined by the commutation relations $\left[\hat{\xi}^{i}, \hat{\xi}^{j}\right]=i \omega^{i j}(\hat{\xi})$, was constructed in [10].
} 
To investigate possible physical implications of dynamical noncommutativity it is reasonable to start with exactly solvable models like the harmonic oscillator and the hydrogen atom and then compare our results with those of canonical noncommutative quantum mechanics [12,13]. For the harmonic oscillator, we suppose that

$$
h(\hat{p}, \hat{q})=\frac{\hat{p}_{i}^{2}}{2}+\frac{\omega_{i}^{2}\left(\hat{q}^{i}\right)^{2}}{2}, \quad V(\hat{\eta})=\frac{\omega_{\eta}^{2} \hat{\eta}^{2}}{2},
$$

so that, after the Bopp shift, the Hamiltonian (11) takes the form

$$
\hat{H}=\frac{\hat{p}_{i}^{2}}{2}+\frac{\omega_{i}^{2}}{2}\left(\hat{x}^{i}-\frac{\theta}{2} f(\hat{\eta}) \varepsilon^{i j} \hat{p}_{j}\right)^{2}+\frac{\hat{p}_{\eta}^{2}}{2}+\frac{\omega_{\eta}^{2}}{2} \hat{\eta}^{2} .
$$

In the coordinate representation, the operators $\hat{x}^{i}=x^{i}$ and $\hat{\eta}=\eta \cdot$ are multiplicative whereas the momenta operators act as derivatives: $\hat{p}_{i}=-i \partial_{i}$ and $\hat{p}_{\eta}=-i \partial_{\eta}$ We employ a perturbation theory over the parameter of noncommutativity $\theta$, starting with the quantum mechanical problem described by the Hamiltonian (14) with $\theta=0$ and then construct corrections for $\theta \neq 0$.

\section{Nonlocality caused by dynamical noncommu- tativity}

It would be interesting to calculate the uncertainty relations between the operators of the noncommutative coordinates $\hat{q}^{i}$, i.e., the nonlocality caused by the dynamical noncommutativity. According to (10) one has:

$$
\left(\triangle q^{1}\right)_{\Psi}\left(\triangle q^{2}\right)_{\Psi} \geq \frac{\theta}{2}|\langle\Psi|f(\hat{\eta})| \Psi\rangle|
$$

where $|\Psi\rangle$ is a given state. If we choose $|\Psi\rangle$ as a stationary state of the system, i.e., an eigenstate of the Hamiltonian (16), it is reasonable to expect that nonlocality will depend on the energy. In general, this is true, however, in some specific models, e.g., $f(\eta)=\eta$ such dependence does not appear.

To calculate the uncertainty relations (17) and the energy spectrum for the quantum system with the Hamiltonian (16) we employ the standard perturbation procedure (see e.g., [14]). Note, however, that the result of this calculation will not depend neither on the choice of basis of operators, nor on the perturbation

method used to solve the problem. First, the hamiltonian (16) is separated into two parts as

$$
\hat{H}=\hat{H}_{0}+\hat{V}
$$

where $\hat{H}_{0}$ is the Hamiltonian of the harmonic oscillator:

$$
\hat{H}_{0}=\frac{\hat{p}_{i}^{2}}{2}+\frac{\omega_{i}^{2}}{2}\left(\hat{x}^{i}\right)^{2}+\frac{\hat{p}_{\eta}^{2}}{2}+\frac{\omega_{\eta}^{2}}{2} \hat{\eta}^{2}
$$


with eigenstates (we use here the notations of creation and annihilation operators, $\left.|n\rangle=\frac{\left(a^{+}\right)^{n}}{\sqrt{n !}}|0\rangle\right)$ :

$$
\left|\Psi_{l m n}^{(0)}\right\rangle=|l\rangle \otimes|m\rangle \otimes|n\rangle
$$

and eigenvalues

$$
E_{l m n}^{(0)}=\omega_{1}\left(l+\frac{1}{2}\right)+\omega_{2}\left(m+\frac{1}{2}\right)+\omega_{\eta}\left(n+\frac{1}{2}\right),
$$

and

$$
\begin{aligned}
& \hat{V}=\hat{V}_{1}+\hat{V}_{2}, \\
& \hat{V}_{1}=\frac{\theta}{2} f(\hat{\eta})\left(\omega_{1}^{2} \hat{x}^{2} \hat{p}_{1}-\omega_{2}^{2} \hat{x}^{1} \hat{p}_{2}\right), \quad \hat{V}_{2}=\frac{\theta^{2} \omega_{i}^{2} \hat{p}_{i}^{2}}{8} f(\hat{\eta})^{2},
\end{aligned}
$$

is the perturbation. We assume that the set $\left|\Psi_{l m n}^{(0)}\right\rangle$ provides an orthonormal basis in the Hilbert space of the system, i.e.,

$$
\left\langle\Psi_{l m n}^{(0)} \mid \Psi_{l^{\prime} m^{\prime} n^{\prime}}^{(0)}\right\rangle=\delta_{l l^{\prime}} \delta_{m m^{\prime}} \delta_{n n^{\prime}}, \quad I=\sum_{l m n}\left|\Psi_{l m n}^{(0)}\right\rangle\left\langle\Psi_{l m n}^{(0)}\right|
$$

To solve the eigenvalue problem for (18), we write:

$$
\begin{aligned}
& \hat{H}|\Psi\rangle=E|\Psi\rangle \\
& |\Psi\rangle=\left|\Psi_{l m n}\right\rangle=\left|\Psi_{l m n}^{(0)}\right\rangle+\left|\Psi_{l m n}^{(1)}\right\rangle+\ldots, \quad E=E^{(0)}+E^{(1)}+\ldots,
\end{aligned}
$$

and express the eigenfunction $\Psi$ in terms of the eigenstates of harmonic oscillator:

$$
\begin{aligned}
\left|\Psi_{l m n}\right\rangle & =\sum_{l^{\prime} m^{\prime} n^{\prime}} C_{l m n}^{l^{\prime} m^{\prime} n^{\prime}}\left|\Psi_{l^{\prime} m^{\prime} n^{\prime}}^{(0)}\right\rangle, \\
C_{l m n}^{l^{\prime} m^{\prime} n^{\prime}} & =C_{l m n}^{(0) l^{\prime} m^{\prime} n^{\prime}}+C_{l m n}^{(1) l^{\prime} m^{\prime} n^{\prime}}+\ldots, \quad C_{l m n}^{(0) l^{\prime} m^{\prime} n^{\prime}}=\delta_{l}^{l^{\prime}} \delta_{m}^{m^{\prime}} \delta_{n}^{n^{\prime}} .
\end{aligned}
$$

From (24) one finds the equation on the coefficients $C_{l m n}^{l^{\prime} m^{\prime} n^{\prime}}$ of the decomposition (25) and eigenvalues $E_{l m n}$ :(here the summation is only over $l^{\prime \prime} m^{\prime \prime} n^{\prime \prime}$ )

$$
\begin{aligned}
& \left(E_{l m n}-E_{l^{\prime} m^{\prime} n^{\prime}}^{(0)}\right) C_{l m n}^{l^{\prime} m^{\prime} n^{\prime}}=\sum_{l^{\prime \prime} m^{\prime \prime} n^{\prime \prime}} V_{l^{\prime \prime} m^{\prime \prime} n^{\prime \prime}}^{l^{\prime} m^{\prime} n^{\prime}} C_{l m n}^{l^{\prime \prime} m^{\prime \prime} n^{\prime \prime}}, \\
& V_{l^{\prime} m^{\prime} n^{\prime}}^{l m n} \equiv\left\langle\Psi_{l m n}^{(0)}|\hat{V}| \Psi_{l^{\prime} m^{\prime} n^{\prime}}^{(0)}\right\rangle=V_{l^{\prime} m^{\prime} n^{\prime}}^{(1) l m n}+V_{l^{\prime} m^{\prime} n^{\prime}}^{(2) l m n},
\end{aligned}
$$

where

$$
\begin{aligned}
& V_{l^{\prime} m^{\prime} n^{\prime}}^{(1) l m n}= \\
& \frac{i \theta}{4 \sqrt{\omega_{1} \omega_{2}}}\left\langle n|f(\hat{\eta})| n^{\prime}\right\rangle\left[( \omega _ { 1 } ^ { 3 } - \omega _ { 2 } ^ { 3 } ) \left(\sqrt{l^{\prime}+1} \sqrt{m^{\prime}+1} \delta_{l, l^{\prime}+1} \delta_{m, m^{\prime}+1}\right.\right. \\
& \left.-\sqrt{l^{\prime} m^{\prime}} \delta_{l, l^{\prime}-1} \delta_{m, m^{\prime}-1}\right)+\left(\omega_{1}^{3}+\omega_{2}^{3}\right)\left(\sqrt{l^{\prime}+1} \sqrt{m^{\prime}} \delta_{l, l^{\prime}+1} \delta_{m, m^{\prime}-1}\right. \\
& \left.\left.-\sqrt{l^{\prime}} \sqrt{m^{\prime}+1} \delta_{l, l^{\prime}-1} \delta_{m, m^{\prime}+1}\right)\right]
\end{aligned}
$$




$$
\begin{aligned}
V_{l^{\prime} m^{\prime} n^{\prime}}^{(2) l m n}= & -\frac{\theta^{2}}{16}\left\langle n\left|f(\hat{\eta})^{2}\right| n^{\prime}\right\rangle\left[\omega _ { 1 } ^ { 3 } \left(\sqrt{\left(l^{\prime}+1\right)\left(l^{\prime}+2\right)} \delta_{l, l^{\prime}+2}-\left(2 l^{\prime}+1\right) \delta_{l, l^{\prime}}(29)\right.\right. \\
& \left.+\sqrt{l^{\prime}\left(l^{\prime}-1\right)} \delta_{l, l^{\prime}-2}\right)+\omega_{2}^{3}\left(\sqrt{\left(m^{\prime}+1\right)\left(m^{\prime}+2\right)} \delta_{m, m^{\prime}+2}\right. \\
& \left.\left.-\left(2 m^{\prime}+1\right) \delta_{m, m^{\prime}}+\sqrt{m^{\prime}\left(m^{\prime}-1\right)} \delta_{m, m^{\prime}-2}\right)\right]
\end{aligned}
$$

Now let us analyze equation (26). It can be solved order by order in $\theta$. In the $n$-th order in $\theta$ this equation with $l=l^{\prime}, m=m^{\prime}$ and $n=n^{\prime}$ gives the $n$-th order correction to the eigenvalue $E$. For arbitrary $l, l^{\prime}, m, m^{\prime}, n, n^{\prime}$, under condition that $E_{l m n}^{(0)}-E_{l^{\prime} m^{\prime} n^{\prime}}^{(0)} \neq 0$, eq. (26) determines the $n$-th order of the coefficients $C_{l m n}^{l^{\prime} m^{\prime} n^{\prime}}$. The $n$-th order of the coefficient $C_{l m n}^{l m n}$ can be determined from the condition of normalization of the state $\left|\Psi_{l m n}\right\rangle$ up to the $n$-th order in $\theta$. Thus, e.g., in the first order in $\theta$ one has:

$$
E_{l m n}^{(1)}=V_{l m n}^{(1) l m n}=0
$$

i.e. the first order correction to energy is equal to zero, like in the case of anharmonic oscillator. The coefficients $C_{l m n}^{l^{\prime} m^{\prime} n^{\prime}}$ in the first order are:

$$
\begin{aligned}
C_{l m n}^{(1) l^{\prime} m^{\prime} n^{\prime}} & =\frac{1}{E_{l m n}^{(0)}-E_{l^{\prime} m^{\prime} n^{\prime}}^{(0)}} V_{l m n}^{(1) l^{\prime} m^{\prime} n^{\prime}}, \forall l^{\prime}, m^{\prime}, n^{\prime} \neq n \\
C_{l m n}^{(1) l^{\prime} m^{\prime} n} & =\frac{1}{E_{l m n}^{(0)}-E_{l^{\prime} m^{\prime} n}^{(0)}} V_{l m n}^{(1) l^{\prime} m^{\prime} n}, \forall l^{\prime}, m^{\prime} \neq m, \\
C_{l m n}^{(1) l^{\prime} m n} & =\frac{1}{E_{l m n}^{(0)}-E_{l^{\prime} m n}^{(0)}} V_{l m n}^{(1) l^{\prime} m n}, \forall l^{\prime} \neq l
\end{aligned}
$$

and

$$
C_{l m n}^{(1) l m n}=0
$$

The second-order corrections to the energy spectrum $E$ and coefficients of the decomposition (25) can be constructed in the same way, but we do not present here the exact formulas.

Let us now calculate expectation value

$$
\begin{aligned}
\left\langle\Psi_{l m n}|f(\eta)| \Psi_{l m n}\right\rangle= & \sum_{l^{\prime} m^{\prime} n^{\prime} n^{\prime \prime}} C_{l m n}^{* l^{\prime} m^{\prime} n^{\prime}} C_{l m n}^{l^{\prime} m^{\prime} n^{\prime \prime}}\left\langle n^{\prime}|f(\eta)| n^{\prime \prime}\right\rangle=\langle n|f(\eta)| n\rangle \\
& +\sum_{n^{\prime}}\left(C_{l m n}^{*(1) l m n^{\prime}}\left\langle n^{\prime}|f(\eta)| n\right\rangle+\left\langle n|f(r)| n^{\prime}\right\rangle C_{l m n}^{(1) l m n^{\prime}}\right) \\
& +\sum_{n^{\prime}}\left(C_{l m n}^{*(2) l m n^{\prime}}\left\langle n^{\prime}|f(\eta)| n\right\rangle+\left\langle n|f(r)| n^{\prime}\right\rangle C_{l m n}^{(2) l m n^{\prime}}\right) \\
& +\sum_{l^{\prime} m^{\prime} n^{\prime} n^{\prime \prime}} C_{l m n}^{*(1) l^{\prime} m^{\prime} n^{\prime}} C_{l m n}^{(1) l^{\prime} m^{\prime} n^{\prime \prime}}\left\langle n^{\prime}|f(\eta)| n^{\prime \prime}\right\rangle+O\left(\theta^{3}\right) .
\end{aligned}
$$


One can easily verify that the first order of this decomposition is equal to zero, because of the special form of the coefficients $C_{l m n}^{(1) l^{\prime} m^{\prime} n^{\prime}}$. The first nontrivial contribution may appear in the second order.

To simplify our considerations we suppose that the system is in the ground state of noncommutativity, $n=0,\left|\Psi_{E}\right\rangle=\left|\Psi_{l m 0}\right\rangle$, and also, $\omega_{\eta} \gg \omega_{1}, \omega_{2}$, i.e., the probability of the system to pass to the first excited state of noncommutativity $(n>0)$ is very small. In what follows we assume that $n=0$ and take into account only the leading powers of the ratio $\omega_{i} / \omega_{\eta}$.

For $f(\eta)=\eta$, the second order contribution in (33) is:

$$
\begin{aligned}
& \sqrt{\frac{n+1}{2 \omega_{\eta}}}\left(C_{l m n}^{*(2) l m n+1}+C_{l m n}^{(2) l m n+1}\right)+\sqrt{\frac{n}{2 \omega_{\eta}}}\left(C_{l m n}^{*(2) l m n-1}+C_{l m n}^{(2) l m n-1}\right) \\
+ & \frac{1}{\sqrt{2 \omega_{\eta}}} \sum_{l^{\prime} m^{\prime} n^{\prime}}\left(\sqrt{n^{\prime}+1} C_{l m n}^{*(1) l^{\prime} m^{\prime} n^{\prime}+1} C_{l m n}^{(1) l^{\prime} m^{\prime} n^{\prime}}+\sqrt{n^{\prime}} C_{l m n}^{*(1) l^{\prime} m^{\prime} n^{\prime}-1} C_{l m n}^{(1) l^{\prime} m^{\prime} n^{\prime}}\right) \\
= & 0 .
\end{aligned}
$$

Thus, for $f(\eta)=\eta$, the uncertainty relations (17) are:

$$
\left(\triangle q^{1}\right)_{\Psi}\left(\triangle q^{2}\right)_{\Psi} \geq O\left(\theta^{4}\right)
$$

i.e., up to high enough order in $\theta$ nonlocality does not appear. So, we can see that noncommutativity does not always lead to nonlocality. However, the energy spectrum in this case changes, the first nontrivial correction being:

$$
E_{l m 0}^{(2)}=\frac{\theta^{2}}{8 \omega_{\eta}}\left[\omega_{1}^{3}\left(l+\frac{1}{2}\right)+\omega_{2}^{3}\left(m+\frac{1}{2}\right)\right] .
$$

Despite the absence of nonlocality, the dynamical noncommutativity with $f(\eta)=$ $\eta$ can contribute to other physical quantities.

For $f(\eta)=\eta^{2}$ in the first order in $\theta$ one has:

$$
\left(\triangle q^{1}\right)_{\Psi}\left(\triangle q^{2}\right)_{\Psi} \geq \frac{\theta}{2 \omega_{\eta}}\left(n+\frac{1}{2}\right)+O\left(\theta^{3}\right) .
$$

Thus, for $n=0$ the right hand side of this expression is just a constant, like in the case of canonical nonlocality but interesting effects can be seen in the higher orders in $\theta$. The second order contribution to (33) is:

$$
\begin{aligned}
& \frac{1}{2 \omega_{\eta}}\left[\sqrt{(n+1)(n+2)}\left(C_{l m n}^{*(2) l m n+2}+C_{l m n}^{(2) l m n+2}\right)\right. \\
& +(2 n+1)\left(C_{l m n}^{*(2) l m n}+C_{l m n}^{(2) l m n}\right)+\sqrt{n(n+1)}\left(C_{l m n}^{*(2) l m n-2}+C_{l m n}^{(2) l m n-2}\right) \\
& +\sum_{l^{\prime} m^{\prime} n^{\prime}}\left(\sqrt{\left(n^{\prime}+1\right)\left(n^{\prime}+2\right)} C_{l m n}^{*(1) l^{\prime} m^{\prime} n^{\prime}+2} C_{l m n}^{(1) l^{\prime} m^{\prime} n^{\prime}}\right. \\
& \left.\left.+\left(2 n^{\prime}+1\right) C_{l m n}^{*(1) l^{\prime} m^{\prime} n^{\prime}} C_{l m n}^{(1) l^{\prime} m^{\prime} n^{\prime}}+\sqrt{n^{\prime}\left(n^{\prime}+1\right)} C_{l m n}^{*(1) l^{\prime} m^{\prime} n^{\prime}-2} C_{l m n}^{(1) l^{\prime} m^{\prime} n^{\prime}}\right)\right] .
\end{aligned}
$$


Now, we put $n=0$ and retain only the second order of the ratio $\omega_{i} / \omega_{\eta}$, we came to

$$
\frac{\theta^{2}}{16 \omega_{1} \omega_{2} \omega_{r}^{2}} \frac{\left(\omega_{1}^{3}-\omega_{2}^{3}\right)^{2}}{\left(\omega_{1}+\omega_{2}\right)^{2}}+\frac{\theta^{2}(2 l m+l+m)}{16 \omega_{1} \omega_{2} \omega_{r}^{2}}\left[\frac{\left(\omega_{1}^{3}-\omega_{2}^{3}\right)^{2}}{\left(\omega_{1}+\omega_{2}\right)^{2}}+\frac{\left(\omega_{1}^{3}+\omega_{2}^{3}\right)^{2}}{\left(\omega_{1}-\omega_{2}\right)^{2}}\right] .
$$

Thus, for the choice $f(\eta)=\eta^{2}$ the uncertainty relations (17) are:

$$
\begin{aligned}
\left(\triangle q^{1}\right)_{\Psi}\left(\triangle q^{2}\right)_{\Psi} \geq & \frac{\theta}{4 \omega_{\eta}}+\frac{\theta^{3}}{32 \omega_{1} \omega_{2} \omega_{\eta}^{2}} \frac{\left(\omega_{1}^{3}-\omega_{2}^{3}\right)^{2}}{\left(\omega_{1}+\omega_{2}\right)^{2}} \\
& +\frac{\theta^{3}(2 l m+l+m)}{32 \omega_{1} \omega_{2} \omega_{\eta}^{2}}\left[\frac{\left(\omega_{1}^{3}-\omega_{2}^{3}\right)^{2}}{\left(\omega_{1}+\omega_{2}\right)^{2}}+\frac{\left(\omega_{1}^{3}+\omega_{2}^{3}\right)^{2}}{\left(\omega_{1}-\omega_{2}\right)^{2}}\right]+O\left(\theta^{4}\right) .
\end{aligned}
$$

In the third order in $\theta$ nonlocality depends on the quantum numbers $l$ and $m$, which determine the zero order energy (21). We have obtained the energy dependent nonlocality, the more the energy of the system, the more the nonlocality (38). Moreover, this contribution is due to the physical degrees of freedom, which correspond to the coordinates $q^{1}$ and $q^{2}$. However, it should be noted that the effect is quite small, comparable to the canonical noncommutativity, since the contribution to the nonlocality is of the $\theta^{3}$ order.

It should be noted that the energy dependent nonlocality also appears in other models of noncommutativity, like kappa-Minkowski space and doubly special relativity; see for review [15. However, it happens in a different manner, through modifications of energy-momentum dispersion relations or energy-dependent generalizations of the spacetime metric [16].

The first nontrivial correction to the energy is:

$$
\begin{aligned}
E_{l m 0}^{(2)}= & \frac{\theta^{2}}{32 \omega_{1} \omega_{2} \omega_{\eta}^{2}}\left[\frac{\left(\omega_{1}^{3}+\omega_{2}^{3}\right)^{2}(l-m)}{\omega_{1}-\omega_{2}}-\frac{\left(\omega_{1}^{3}-\omega_{2}^{3}\right)^{2}(l+m+1)}{\omega_{1}+\omega_{2}}\right] \\
& +\frac{3 \theta^{2}}{64 \omega_{\eta}^{2}}\left[\omega_{1}^{3}\left(l+\frac{1}{2}\right)+\omega_{2}^{3}\left(m+\frac{1}{2}\right)\right] .
\end{aligned}
$$

Note that this quantity is of the order $\left(\omega_{i} / \omega_{\eta}\right)^{2}$, while the correction to the energy (35) is of the order $\omega_{i} / \omega_{\eta}$.

Note that if we choose $f(\eta)=\eta^{2}-1 / \omega_{\eta}$, the expression (38) will have no contribution in the first order in $\theta$, first nontrivial contribution will appear only in $\theta^{3}$ order. That is in this case the contribution of the physical degrees of freedom to nonlocality is significant. Thus, by choosing $f(\eta)$ adequately we may control nonlocality, accordingly to physical considerations.

\section{Conclusion}

In conclusion we note that the proposed model of noncommutativity is in fact a generalization of canonical noncommutativity $\left[\hat{x}^{i}, \hat{x}^{j}\right]=i \theta^{i j}$, which can be ob- 
tained just by taking $f(\eta)=$ const. However, dynamical noncommutativity provides much more freedom in the description of the physical effects caused by noncommutativity, connected with the presence of one more degree of freedom. One interesting possibility would be to consider this degree of freedom as an effective gravitational degree of freedom, which may correspond to a compact extra dimension, like in Kaluza - Klein theory of gravity, or to an extended extra dimension, like in Randall - Sundrum model.

We have discussed two different choices of theinteraction, i.e., the function $f(\eta)$. In the first case, $f(\eta)=\eta$, the nonlocality does not show up to quite higher orders in $\theta$, see (34), while in the second one, $f(\eta)=\eta^{2}$, nonlocality appears already in the first order in $\theta$, see (36) and (38). Thus, choosing the functions $V(r)$ and $f(r)$ from some physical considerations we are able to control noncommutativity, nonlocality and its contribution to physical quantities like the energy spectrum, etc.

\section{Acknowledgements}

We are gratefull to Prof. H.O. Girotti and to Dr. F.S. Bemfica for very usefull discussions. V.G.K. thanks the Institute of Physics of Universidade Federal do Rio Grande do Sul for hospitality. V.G.K. acknowledges FAPESP for support. M.G. and A.J.S. thank FAPESP and CNPq for partial support.

\section{References}

[1] S. Doplicher, K. Fredenhagen and J. Roberts, Commum.Math.Phys. 172 (1995) 187.

[2] N. Seiberg and E. Witten, JHEP 9909 (1999) 032; M. Douglas, N. Nekrasov, Rev.Mod.Phys.73 (2001) 977-1029; R. Szabo, Phys.Rept.378 (2003) 207299.

[3] C. Duval, P.A. Horvathy, Phys.Lett.B479 (2000) 284; J. Gamboa, M. Loewe and J.C. Rojas, Phys.Rev.D 64 (2001) 067901; S. Bellucci, A. Nersessian, C. Sochichiu, Phys.Lett.B522 (2001) 345; A.F. Ferrari, M. Gomes, C.A. Stechhahn, Phys.Rev.D76 (2007) 085008; P.G. Castro, B. Chakraborty, F. Toppan, J.Math.Phys. 49 (2008) 082106; F.S. Bemfica, H.O. Girotti, Phys.Rev.D77 (2008) 027704, Phys.Rev.D79 (2009) 125024; H. Falomir, J. Gamboa, J. Lopez-Sarrion, F. Mendez, P.A.G. Pisani, Phys.Lett.B680 (2009) 384 .

[4] R. Szabo, Class.Quant.Grav.23 (2006) R199-R242.

[5] M. Gomes, V.G. Kupriyanov, Phys.Rev.D79 (2009) 125011. 
[6] G. Dunne, R. Jackiw, C.A. Trugenberger, Phys.Rev.D41 (1990) 661.

[7] A.A. Deriglazov, Phys.Lett. B555 (2003) 83, D.M. Gitman and V.G. Kupriyanov, Eur.Phys.J. C 54 (2008) 325.

[8] J. Lukierski, P.C. Stichel., W.J. Zakrzewski, Annals Phys.260 (1997) 224249 .

[9] P.A. Horvathy, M.S. Plyushchay, JHEP06 (2002) 033.

[10] V.G. Kupriyanov, D.V. Vassilevich, Eur.Phys.J.C. 58 (2008) 627-637.

[11] F. Bopp, Ann. Inst. Henri Poincare 15 (1956), 81.

[12] M. Chaichian, M.M. Sheikh-Jabbari and A. Tureanu, Phys.Rev.Lett. 86 (2001) 2716; T.C. Adorno, M.C. Baldiotti, M. Chaichian, D.M. Gitman, A. Tureanu, Dirac Equation in Noncommutative Space for Hydrogen Atom, arXiv:0904.2836 [hep-th].

[13] V.P. Nair, A.P. Polychronakos, Phys.Lett.B505 (2001) 267-274; C. Duval, P.A. Horvathy, J.Phys.A34 (2001) 10097-10108.

[14] L.D. Landau, E.M. Lifshits, Quantum mechanics, Oxford:Pergamon, 1962.

[15] J. Kowalski-Glikman, Lect.Notes Phys.669 (2005) 131-159.

[16] G. Amelino-Camelia, M. Arzano, Yi Ling, G. Mandanici, Class.Quant.Grav.23 (2006) 2585-2606. 